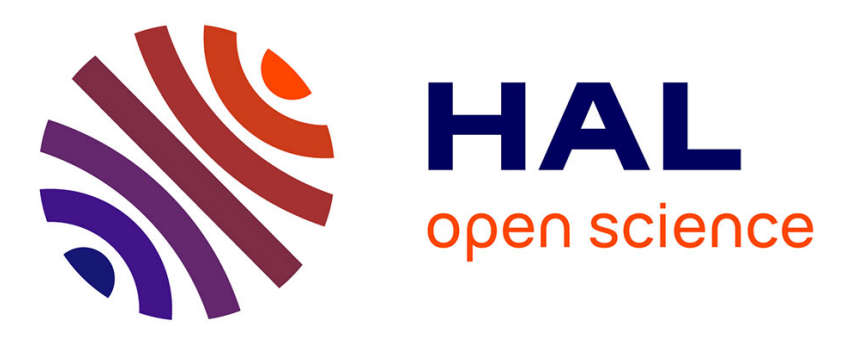

\title{
Clostridium difficile infection in acute flares of inflammatory bowel disease: A prospective study
}

Harry Sokol, Valérie Lalande, Cecilia Landman, Anne Bourrier, Isabelle

Nion-Larmurier, Sylvie Rajca, Julien Kirchgesner, Philippe Seksik, Jacques

Cosnes, Frédéric Barbut, et al.

\section{To cite this version:}

Harry Sokol, Valérie Lalande, Cecilia Landman, Anne Bourrier, Isabelle Nion-Larmurier, et al.. Clostridium difficile infection in acute flares of inflammatory bowel disease: A prospective study.

Digestive and Liver Disease, 2017, 49 (6), pp.643-646. 10.1016/j.dld.2017.01.162 . hal-01472374

\section{HAL Id: hal-01472374 \\ https://hal.sorbonne-universite.fr/hal-01472374}

Submitted on 20 Feb 2017

HAL is a multi-disciplinary open access archive for the deposit and dissemination of scientific research documents, whether they are published or not. The documents may come from teaching and research institutions in France or abroad, or from public or private research centers.
L'archive ouverte pluridisciplinaire HAL, est destinée au dépôt et à la diffusion de documents scientifiques de niveau recherche, publiés ou non, émanant des établissements d'enseignement et de recherche français ou étrangers, des laboratoires publics ou privés. 


\section{Clostridium difficile infection in acute flares of inflammatory bowel disease: a prospective study}

Running head: CDI diagnosis in IBD

Harry Sokol ${ }^{1,2,3,4,{ }^{*}}$, Valérie Lalande ${ }^{5}$, Cecilia Landman ${ }^{1}$, Anne Bourrier ${ }^{1}$, Isabelle Nion-Larmurier ${ }^{1}$, Sylvie Rajca $^{1}$, Julien Kirchgesner ${ }^{1}$, Philippe Seksik ${ }^{1}$, Jacques Cosnes ${ }^{1}$, Frédéric Barbut ${ }^{5,6}$, Laurent Beaugerie ${ }^{1}$

${ }^{1}$ Department of Gastroenterology, Saint Antoine Hospital, AP-HP, and GRC-UPMC 03, UPMC University Paris 06, Paris, France

${ }^{2}$ Sorbonne University - UPMC Univ Paris 06, INSERM ERL 1157, Avenir Team Gut Microbiota and Immunity, UMR 7203, Saint-Antoine Hospital, Paris, France

${ }^{3}$ INRA, UMR1319 Micalis \& AgroParisTech, Jouy en Josas, France

${ }^{4}$ Inflammation-Immunopathology-Biotherapy Department (DHU i2B), Paris, France

${ }^{5}$ Department of Microbiology, Saint Antoine Hospital, AP-HP, UPMC University Paris 06, Paris, France

${ }^{6}$ National Reference Laboratory for Clostridium difficile, Saint Antoine Hospital, AP-HP, UPMC University Paris 06, Paris, France

${ }^{7}$ Clinical research group EPIDIFF, UPMC University Paris 03, Paris, France

*Corresponding author: Harry Sokol, Service de Gastroentérologie et Nutrition, Hôpital Saint-Antoine, 184 rue du faubourg St Antoine, 75571 Paris cedex 12, France. E-mail: harry.sokol@aphp.fr Phone: +33149283 162. Fax: +33149283188 


\begin{abstract}
Objectives

Clostridium difficile infection (CDI) is a common complication in inflammatory bowel disease (IBD) and has been associated with poor IBD outcome. The aims of our study were to look for predictive factors of CDI in patients hospitalized for IBD flare and to evaluate a rapid testing strategy in this population.
\end{abstract}

Methods

Consecutive patients hospitalized for IBD flare in Saint-Antoine Hospital (Paris, France) were prospectively tested for CDI with a defined strategy involving rapid testing and reference methods. Risk factors for CDI were investigated and performances of diagnostic tests were evaluated.

Results

C. difficile testing was performed at admission in 461 hospitalizations for IBD flare. CDI was diagnosed in 35 cases (7.6\%) and non-toxigenic $C$. difficile was identified in 10 cases (2.2\%). In multivariate analysis, UC phenotype was associated with CDI (OR 2.2, 95\% Cl 1.03-4.6, p=0.047). Glutamate dehydrogenase $(\mathrm{GDH})$ test had a $97.1 \%$ sensitivity and a $100 \%$ negative predictive value for CDI diagnosis but a positive predictive value of 79.1\%. Enzyme immunoassay (EIA)-based toxin detection (C. Diff Quik Chek complete ${ }^{\circledR}$, Alere) had a poor sensitivity and diagnosis was rescued by toxin PCR in $100 \%$ of cases.

Conclusion

CDI is frequent in patients hospitalized for IBD flare. Clinical parameters do not help for the diagnosis and rapid testing should be performed in all patients. Currently, a negative result of an EIA-based toxin search associated with a positive GDH test cannot rule out a CDI and should not delay initiation of specific treatment in case of severe symptoms or high presumption.

Word count: 1723 
Keywords: crohn; ulcerative colitis; Clostridium difficile

\section{Introduction}

Clostridium difficile is a ubiquitous anaerobic spore-forming Gram-positive bacterium. Only toxigenic strains have a pathogenic potential and approximately $3 \%$ of healthy adults are asymptomatic carriers of $C$. difficile (1). Beside post-antibiotics and nosocomial settings, $C$. difficile infection (CDI) has been repeatedly associated with inflammatory bowel disease (IBD). Indeed, as an imbalance of the gut microbiota composition (i.e. dysbiosis) is present in patients with IBD (2), a CDI can occur even without antibiotics trigger. Although risk factors of CDI in IBD have been poorly investigated, some authors found that ulcerative colitis (compared to Crohn's disease) and more generally colonic involvement were associated with $\operatorname{CDI}(3,4)$. In a previous retrospective study, we observed that intake of nonsteroidal anti-inflammatory drugs (NSAIDs) within the two months before admission for IBD flare was a risk factor for CDI (5). Importantly, CDI has been associated with poor IBD outcome with increased severity of relapse, longer hospital stay, higher rate of colectomy and mortality $(4,6-8)$. Moreover, toxin-producing CDI can trigger or worsen an IBD flare and the diagnosis should thus be done as soon as possible in order to start the appropriate treatment. The early diagnosis of CDI in a patient with IBD is thus very important, but is particularly difficult as clinical symptoms of CDI cannot be distinguished from those of an IBD flare. Gold standard tests such as the stool cytotoxicity assay and toxigenic culture are labor-intensive and require specific laboratory facilities and technical expertise, limiting their widespread use. Moreover, these methods are time-consuming, necessitating several days to get a result and are thus not appropriate when an urgent diagnosis is required. On the other hand, enzyme immunoassays (EIAs) for glutamate dehydrogenase (GDH) and toxins $A$ and $B$ are easier, faster and cheaper. GDH-based assay is known to have a good sensitivity but does not allow discrimination between toxigenic and non-toxigenic strains. Conversely, EIAs for toxins have a low 
sensitivity and are thus not recommended as a standalone detection method $(9,10)$. The efficacy of these diagnostic methods in IBD populations is not well characterized.

The aims of our prospective study were to look for clinical predictive factors of $C D I$ in patients hospitalized for IBD flare and to evaluate a rapid testing strategy in this population.

\section{Patients and Methods}

Study population

All consecutive patients with IBD hospitalized for flare in the Gastroenterology department of the Saint Antoine Hospital (Paris, France) from September 2012 to May 2014, were prospectively tested for $C$. difficile infection. Clinical data were obtained from the MICISTA registry, a prospective clinical database of all IBD patients evaluated by the same physicians at Saint Antoine Hospital. Information regarding treatment at admission was obtained from medical records. Beside treatments for IBD, we specifically recorded the use of antibiotics, NSAIDs and proton-pump inhibitors (PPI) within two months before admission.

\section{C. difficile testing}

Diagnosis of CDI was performed using a stool culture on selective medium (TCCA, taurocholate cycloserine cefoxitine medium) and a stool cytotoxicity assay on MRC-5 cells. For stool cytotoxicity assay Fresh stool specimens were diluted in phosphate-buffered saline buffer (1:10 [wt/vol]) and centrifuged at 2,500 g for $30 \mathrm{~min}$. The supernatant was passed through a $0.45-\mathrm{m}$-pore-size filter and inoculated onto confluent monolayers of MRC-5 cells in 96-well microtiter plates that were incubated at $37^{\circ} \mathrm{C}$ in a $6.5 \% \mathrm{CO} 2$ atmosphere for $48 \mathrm{~h}$. The final dilution of the fecal filtrate in each well was 1:100. Samples were considered positive if a characteristic cytopathic effect (cell rounding) was observed for 
at least $50 \%$ of the cells and could be neutralized with anti-Clostridium sordellii antiserum (11). In case of positive culture and negative stool cytotoxicity assay, a toxigenic culture (determination of the ability of the isolate to produce toxins in vitro) was performed as previously described (11). Culture was performed on selective medium (brain heart infusion broth supplemented with $5 \%$ defibrinated horse blood, $0.1 \%$ taurocholate, $250 \mathrm{~g} / \mathrm{ml}$ cycloserine, and $10 \mathrm{~g} / \mathrm{ml}$ cefoxitin), and plates were incubated for $48 \mathrm{~h}$ in an anaerobic atmosphere. Colonies were identified by use of an enzymatic profile from the RapID32A gallery (bioMe'rieux, La Balme les Grottes, France). Then, $C$. difficile isolates were incubated in brain heart infusion broth for 5 days, and the supernatant was tested using the cytotoxicity assay (11).

All 461 samples were also tested for C. difficile-specific Glutamate dehydrogenase (GDH) by Enzyme immunoassay (EIA) and 168 samples were tested for free toxin A and B by EIA method (C. Diff Quik Chek complete ${ }^{\circledR}$, Alere) according to manufacturer instructions. In case of discrepancy between GDH and toxins, PCR targeting toxins (Gen Xpert C. difficile, Cepheid) and enriched culture were performed. CDI was defined by a clinical picture compatible with CDI (diarrhoea or ileus) and microbiological evidence of toxin-producing Clostridium difficile in stools, either by a positive toxigenic culture or a positive stool cytotoxicity assay.

\section{Statistical analysis}

The results of the quantitative variables are expressed as median and range. The qualitative variables are expressed as numbers and percentages. To identify the predictive factors of CDI, a univariate analysis was performed using the Chi2test, Fisher's exact test and t test when appropriate. The variables taken into account were: age, gender, type of IBD, duration of disease, current smoking status, medical history (intestinal resection, PSC, appendectomy), disease activity in the previous 2 years, IBD treatment (corticosteroid equivalent dose equal to or greater than 20 milligrams of prednisolone, immunosuppressants, anti-TNF $\alpha$ ) or other treatment (PPI, antibiotics, NSAIDs) in the two months prior to admission. In case of multiple positive samples in the same patient, only the first 
one was taken into account. In case of multiple negative samples in the same patient, only samples with at least 3 months interval were taken into account. A p value of less than 0.05 was considered statistically significant. The variables with $p$ values $<0.20$ in the univariate analysis entered a multivariate logistic regression analysis using a backward stepwise procedure. The data were processed using JMP ${ }^{\circledR}$ software (version 7.0,SAS Institute Inc., Cary, NC).

\section{Results}

Characteristics of the study population

Between September 2012 and May 2014, C. difficile testing was performed at admission in 461 hospitalizations for IBD flare in 342 patients. One hundred and six patients were hospitalized at least twice during the study period. Clinical characteristics of the patients are indicated in Table 1 . As expected, active smoking and history of appendectomy were more frequent in $C D$. Patients with $C D$ were more frequently treated by anti-TNF and immunomodulators. Two thirds of the CD patients had colon involvement ( $\mathrm{L} 2$ and $\mathrm{L} 3$ of the Montreal classification).

Clostridium difficile infection rate and predictors

Among the 461 hospitalizations for IBD flare, $C$. difficile infection was diagnosed in 35 cases $(7.6 \%)$ and non-toxigenic $C$. difficile was identified in 10 cases (2.2\%). Among patients with CDI, 12 (34.3\%) had free toxins in stools whereas $23(65.7 \%)$ harbored a toxigenic strain without detectable toxins by the stool cytotoxicity assay. In univariate analysis, among the variables taken into account, history of appendectomy had a protective effect whereas UC was associated with a higher risk of CDI (Table 2). A trend was observed for a protective effect of smoking and appendectomy and a promoting effect of corticosteroid treatment. No effect was noted for colon involvement, anti-TNF or immunomodulators, 
NSAIDs or PPI use. In multivariate analysis, the association between UC and a higher risk of CDI remained statistically significant (OR $2.2,95 \% \mathrm{Cl} 1.03-4.6)$.

Accuracy of rapid $C$. difficile testing in IBD patients

The sensitivity, specificity, positive (PPV) and negative (NPV) predictive values of EIA for GDH and EIA for toxins (Alere) for the diagnosis of $C$. difficile infection are presented in table 3. GDH alone had a $100 \%$ NPV for the diagnosis of CDI but its PPV was below $80 \%$ as the test does not discriminate between toxigenic and non-toxigenic strains. Conversely, EIA for toxins A/B had a poor sensitivity (18.2\%). However, PPV and specificity were excellent. Among the false negative cases of the Tox A/BEIA (GDH positive / toxin negative), diagnosis was rescued by toxin PCR in 100\% of cases and by toxigenic culture in $88.9 \%$ of cases. Importantly, stool cytotoxicity assay was negative in all these cases of GDH positive/toxin negative Tox a/b Quik chek ${ }^{\circledR}$ results.

\section{Discussion}

In this prospective study, we showed that Clostridium difficile infection (CDI) is a frequent feature in patients hospitalized for IBD flare, reaching almost $8 \%$. After multivariate analysis, the only predictive factor of $C D I$ was the UC (vs $C D$ ) phenotype. Interestingly, in univariate analysis, smoking and appendectomy, two protective factors regarding UC had also a protective effect for CDI. Despite the acknowledged role of PPIs in CDI (12) and their possible role in IBD flare (13), PPIs were not associated with $\mathrm{CDI}$ in the current study.

Finally, we showed that rapid testing is feasible in IBD patient populations.

CDI is a well-known complication in IBD. Several studies suggested that UC phenotype, and more generally colon involvement, are risk factors for $\operatorname{CDI}(3,4)$. Consequently, screening for $C$. difficile has 
been recommended at every flare in patients with colonic disease by ECCO consensus (14). In our study, although UC phenotype was identified as a risk factor for CDI, colon involvement by itself was not. The risk of CDI infection in patients without colon involvement was $7.0 \%$ ( 5 cases out of 71 ). Moreover, the risk of CDI was still high in $\mathrm{CD}$ patients ( $5.5 \%$ vs $11.3 \%$ in UC) supporting the need for systematic screening in CD as well. In accordance with other studies (15), we observed a trend for an association between corticosteroid treatment and CDI. This association was not statistically significant after multivariate analysis, possibly because of insufficient statistical power. Contrary to other studies (10), we did not observe any effect of immunomodulators on CDI risk. Effect of NSAIDs could not be properly evaluated as their use was very limited in our study population ( 7 patients in the whole population). Our study has several limitations including insufficient sample size to detect factors with small effect on CDI risk and the impossibility to evaluate the long term effect of CDI on IBD outcome.

One of the limitations of our study is that it is performed in a single tertiary centre, and thus the results might not be generalizable.

The high frequency of $\mathrm{CDI}$ in patients hospitalized for IBD flare and its potential severity in IBD populations require a strategy to rapidly test for $\mathrm{CDI}$ and start an appropriate treatment. In the current study, we confirm that glutamate deshydrogenase (GDH) has an excellent NPV in IBD populations allowing to rule out $\mathrm{CDI}$ diagnosis if negative. On the other hand, EIA-based toxin search had a poor sensitivity when compared to nucleic acid-based method or gold standard methods such as toxigenic culture. These results show that EIA-based toxin testing is not appropriate for a standalone diagnosis test in IBD populations.

Interestingly, stool cytotoxicity assay was negative in all cases of false negative EIA-based toxin search results. The significance of a positive stool culture with negative stool cytotoxicity assay remains questionable. If PCR for toxin genes or toxigenic culture is positive, the diagnosis of CDI is accepted. However PCR detects genes, and thus the theoretical ability of the strain to produce toxins but not production itself. The toxigenic culture detects strains that can produce toxins in vitro but a positive 
result does not mean that there is in vivo production in the patient. Indeed, recent studies in general population and single center studies suggest that detection of toxigenic $C$. difficile strains by PCR in the absence of free toxin in the stools does not influence clinical outcome $(10,16,17)$. This question is still actively debated and the current study was not designed to address it. Further specific studies are thus required to solve this issue. Meanwhile, classical diagnosis criteria should be used and EIA-based tests are thus not sensitive enough to be used alone in IBD populations. Based on our results, it can be recommended to test all patients hospitalized for IBD flare with GDH and EIA-based toxin search. If GDH is negative, $\mathrm{CDI}$ can be reasonably ruled out. If GDH is positive but EIA-based toxin search is negative, a confirmation test (PCR for toxin genes and/or toxigenic culture) should be performed. In the case of severe symptoms and/or high presumption, specific antibiotics should be started before getting the results of these confirmation tests.

In conclusion, $\mathrm{CDI}$ is frequent in patients hospitalized for IBD flare. Clinical parameters do not help for the diagnosis and rapid testing should be performed in all patients. Currently, a negative result of an EIA-based toxin search associated with a positive GDH test cannot rule out a CDI and should not delay initiation of specific treatment in case of severe symptoms or high presumption.

\section{Statement of Interests}

\section{Authors' declaration of personal interests:}

HS received consulting fee from Danone, Enterome, Astellas, Roche, Merk and lecture fee from Abbvie and Biocodex and Takeda.

LB has received consulting fees from Abbott and Janssen, lecture fees from Abbott, Abbvie, MSD, Ferring Pharmaceuticals, and research support from Abbott, Biocodex and Ferring Pharmaceuticals PS received consulting fees from Abbvie, Merck-MSD and Biocodex, grants from Biocodex, sponsored travel from Merck-MSD and Takeda 
$J C$ received consulting fee from VIFOR Pharma, lecture fee Abbvie

$\mathrm{CL}$ received travelling support from Abbvie and Biocodex.

FB received consulting fee from Danone, Astellas, MSD, Pfizer, Sanofi Pasteur ad lecture fee from MSD, Astellas.

Declaration of funding interests :

This study was supported by the French society of gastroenterology (FARE grant) and by a grant from Biocodex.

Authorship

Statement:

Guarantor

of

the

article:

Harry

Sokol

Specific author contributions: HS and LB conceived and designed the study. HS performed data analysis and wrote the manuscript. VL performed microbiology analysis and testing. $H S, C L, A B, I N L$, SR, JK, PS, JC, LB provided data and samples for the IBD patients. All of the authors discussed the results and improved the manuscript.

All authors approved the final version of the manuscript. 


\section{References}

1. Natarajan M, Walk ST, Young VB, et al. A clinical and epidemiological review of non-toxigenic Clostridium difficile. Anaerobe 2013;22:1-5.

2. Sokol H, Lay C, Seksik P, et al. Analysis of bacterial bowel communities of IBD patients: What has it revealed? Inflamm Bowel Dis 2008;14:858-867.

3. Issa M, Vijayapal A, Graham MB, et al.Impact of Clostridium difficile on inflammatory bowel disease. Clin Gastroenterol Hepatol 2007;5:345-51.

4. Rodemann JF, Dubberke ER, Reske KA, et al. Incidence of Clostridium difficile infection in inflammatory bowel disease. Clin Gastroenterol Hepatol 2007;5:339-44.

5. Regnault $\mathrm{H}$, Bourrier $\mathrm{A}$, Lalande $\mathrm{V}$, et al. Prevalence and risk factors of Clostridium difficile infection in patients hospitalized for flare of inflammatory bowel disease: a retrospective assessment. Dig Liver Dis 2014;46:1086-92.

6. Ananthakrishnan AN, McGinley EL, Binion DG. Excess hospitalisation burden associated with Clostridium difficile in patients with inflammatory bowel disease. Gut 2008;57:205-10.

7. Ananthakrishnan AN, McGinley EL, Saeian K, et al. Temporal trends in disease outcomes related to Clostridium difficile infection in patients with inflammatory bowel disease. Inflamm Bowel Dis 2011;17:976-83.

8. Nguyen GC, Kaplan GG, Harris ML, et al. A national survey of the prevalence and impact of Clostridium difficile infection among hospitalized inflammatory bowel disease patients. Am J Gastroenterol 2008;103:1443-50.

9. Crobach MJ, Dekkers OM, Wilcox MH, et al. European Society of Clinical Microbiology and Infectious Diseases (ESCMID): data review and recommendations for diagnosing Clostridium difficile-infection (CDI). Clin Microbiol Infect 2009;15:1053-66.

10. Leffler DA, Lamont JT. Clostridium difficile infection. N Engl J Med 2015;372:1539-48.

11. Barbut F, Braun M, Burghoffer B, et al. Rapid detection of toxigenic strains of Clostridium difficile in diarrheal stools by real-time PCR. J Clin Microbiol 2009;47:1276-7. 
12. Debast SB, Bauer MP, Kuijper EJ et al. European Society of Clinical Microbiology and Infectious Diseases: update of the treatment guidance document for Clostridium difficile infection. Clin Microbiol Infect. 2014 Mar;20 Suppl 2:1-26.

13. Juillerat $\mathrm{P}, \mathrm{Schneeweiss} \mathrm{S}$, Cook EF et al. Drugs that inhibit gastric acid secretion may alter the course of inflammatory bowel disease. Aliment Pharmacol Ther. 2012 Aug;36(3):239-47.

14. Rahier JF, Magro F, Abreu C et al. European Crohn's and Colitis Organisation (ECCO). Second European evidence-based consensus on the prevention, diagnosis and management of opportunistic infections in inflammatory bowel disease. J Crohns Colitis. 2014 Jun;8(6):443-68.

15. Schneeweiss S, Korzenik J, Solomon DH, et al. Infliximab and other immunomodulating drugs in patients with inflammatory bowel disease and the risk of serious bacterial infections. Aliment Pharmacol Ther 2009;30:253-64.

16. Planche TD, Davies KA, Coen PG, et al.Differences in outcome according to Clostridium difficile testing method: a prospective multicentre diagnostic validation study of $\mathrm{C}$ difficile infection. Lancet Infect Dis 2013;13:936-45.

17. Polage CR, Gyorke CE, Kennedy MA, et al.Overdiagnosis of Clostridium difficile Infection in the Molecular Test Era. JAMA Intern Med 2015;175:1792-801. 


\section{Tables}

Table 1 : Characteristics of patients included in the study

\begin{tabular}{|c|c|c|c|}
\hline & Crohn's disease & Ulcerative colitis & $p$ \\
\hline$n$ & 219 & 123 & \\
\hline Male gender, n (\%) & $91(41.6)$ & $63(51.2)$ & 0.09 \\
\hline Age (year) Median (range) & $34(17-88)$ & $37(20-90)$ & 0.25 \\
\hline Active smoking, $\mathrm{n}(\%)$ & $84(38.4)$ & $13(10.6)$ & $<0.0001$ \\
\hline Duration of disease (year) Median (range) & $10(0-50)$ & $5(0-58)$ & $<0.0001$ \\
\hline Prior Intestinal resection, $\mathrm{n}(\%)$ & $102(46.6)$ & $22(17.9)$ & $<0.0001$ \\
\hline Appendectomy, n (\%) & $50(22.8)$ & $9(7.3)$ & 0.0001 \\
\hline PSC, n (\%) & $4(1.8)$ & $6(4.9)$ & 0.11 \\
\hline Active disease in the 2 years before, $n(\%)$ & $99(45.2)$ & $55(44.7)$ & 0.99 \\
\hline Anti-TNF treatment, $\mathrm{n}(\%)$ & $75(34.2)$ & $28(22.8)$ & 0.02 \\
\hline Thiopurine/MTX treatment, $\mathrm{n}(\%)$ & $71(32.4)$ & $27(22)$ & 0.04 \\
\hline Corticosteroids, n (\%) & $36(16.4)$ & $25(20.3)$ & 0.34 \\
\hline PPI, n (\%) & $23(10.5)$ & $13(10.6)$ & 0.96 \\
\hline NSAIDs, n (\%) & $1(0.5)$ & $3(2.4)$ & 0.11 \\
\hline Antibiotics, n (\%) & $34(15.5)$ & $13(10.6)$ & 0.21 \\
\hline Montreal classification & L1 71 (32.4) & E1 $9(7.3)$ & \\
\hline
\end{tabular}


L2 $56(25.6)$

L3 88 (40.2)

L4 35 (16)

p $100(45.7)$
E2 46 (37.4)

E3 $68(55.3)$

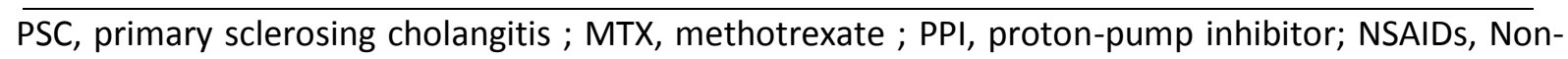
steroidal anti-inflammatory drugs 
Table 2 : Characteristics of patients with or without CDI

\begin{tabular}{|c|c|c|c|}
\hline & $C D I+(n=30)$ & $C D I-(n=364)$ & $\mathrm{p}$ \\
\hline Male gender, n (\%) & $16(53.3)$ & $150(41.2)$ & $0.20 \#$ \\
\hline $\mathrm{CD} / \mathrm{UC}, \mathrm{n}(\%)$ & $14(46.7) / 16$ (53.3) & $239(65.7) / 125$ (34.3) & $0.04 \#$ \\
\hline Appendectomy, n (\%) & 0 & $64(17.6)$ & $0.008 \#$ \\
\hline Active smoking, $\mathrm{n}(\%)$ & $4(13.3)$ & $103(28.3)$ & $0.06 \#$ \\
\hline Small intestine involvement, $\mathrm{n}(\%)$ & $13(43.3)$ & $189(51.9)$ & 0.37 \\
\hline Perianal lesions, n (\%) & $8(26.7)$ & $117(32.1)$ & 0.53 \\
\hline colon involvement, $\mathrm{n}(\%)$ & $25(83.3)$ & $294(80.8)$ & 0.73 \\
\hline pancolitis, n (\%) & $9(30.0)$ & $117(32.1)$ & 0.81 \\
\hline PSC, n (\%) & $2(6.7)$ & $11(3.0)$ & 0.34 \\
\hline Prior Intestinal resection, n (\%) & $8(26.7)$ & $137(37.1)$ & 0.24 \\
\hline Active disease in the 1 years before, $n(\%)$ & $12(40)$ & $134(36.8)$ & 0.73 \\
\hline Duration of disease (year) Median (range) & $5(0-24)$ & $8(0-58)$ & $0.18 \#$ \\
\hline Age (year) Median (range) & $34(20-79)$ & $35(17-90)$ & 0.58 \\
\hline Anti-TNF treatment, $\mathrm{n}(\%)$ & $8(26.7)$ & $118(32.4)$ & 0.51 \\
\hline Thiopurine/MTX treatment, $\mathrm{n}(\%)$ & $6(20.0)$ & $115(31.6)$ & $0.17 \#$ \\
\hline Corticosteroids, n (\%) & $9(30.0)$ & $60(16.6)$ & $0.08 \#$ \\
\hline PPI, n (\%) & $2(6.7)$ & $41(11.3)$ & 0.41 \\
\hline NSAIDs, n (\%) & 0 & $7(1.9)$ & 1 \\
\hline Antibiotics, n (\%) & $6(20.0)$ & 47 (12.9) & 0.30 \\
\hline
\end{tabular}

\# : included in multivariate analysis $(p<0.2)$

In case of multiple positive samples in the same patient, only the first one was taken into account (5 samples were in this case).

In case of multiple negative samples in the same patient, only samples with at least 3 months interval were taken into account (62 samples were in this case) 
Table 3: Sensitivity, specificity, PPV and NPV of Quik chek for diagnosis of $C$. difficile infection

\begin{tabular}{llllll}
\hline & Sensitivity & Specificity & & & \\
& $(\%)$ & $(\%)$ & PPV & NPV & Accuracy \\
\hline GDH (considered alone) & $97.1 \%$ & $97.9 \%$ & $79.1 \%$ & $100 \%$ & $97.6 \%$ \\
EIA for toxins A/B & $18.2 \%$ & $100 \%$ & $100 \%$ & $94.5 \%$ & $95.2 \%$ \\
\hline
\end{tabular}

